\title{
CONTINUIDADES Y DISCONTINUIDADES EN LA ENSEÑANZA DE LA MATEMÁTICA DE TRES GENERACIONES. ESTUDIO DE CASO: SEXTO AÑO DE ESTUDIO EN UNA ESCUELA PRIMARIA
}

\author{
Marcel David Pochulu \\ Universidad Nacional de Villa María, Argentina
}

\section{INTRODUCCIÓN}

En muchas ocasiones, quienes somos docentes y estamos en educación, sentimos la vaga sensación que todo tiempo pasado fue mejor. Que ayer era más seguro que hoy, que se veían las cosas de otra forma y que nuestros alumnos eran mejores, más estudiosos y con más ganas de aprender. Pero no es posible regresar el tiempo atrás, no podemos y no debemos volver sobre lo andado, ni escribir sobre lo escrito, a pesar que nuestros corazones nos impulsen a decir "iTodo tiempo pasado fue mejor!".

En este sentido, el trabajo que presentamos a continuación, analiza la enseñanza de la matemática del sexto año de estudio de la educación primaria argentina, en una escuela particular, y desde un pasado no muy lejano hasta un presente muy cercano. Como objetivo general nos planteamos:

Analizar las continuidades y discontinuidades que presentó la enseñanza de la matemática, para tres generaciones - abuelo, padre e hijo - que asistieron a una misma escuela primaria.

Para ello, hemos analizado los cuadernos y carpetas de clases de matemática, que fueron utilizados por los integrantes de esta familia durante los años 1955, 1978 y 2002.

\subsection{Contexto de la investigación}

Hablar de prácticas educativas, en cualquier campo, requiere una mención especial al contexto social donde ésta se desarrolla, ya que la estructura de la sociedad y los grupos que la conforman determinan las condiciones para las mismas y los objetivos que ellas persiguen.

Las prácticas de enseñanza en matemática no son ajenas a dicha relación, en tanto los objetos del conocimiento matemático son construcciones sociales que se recrean constantemente en el proceso de interacción entre los conocimientos matemáticos individuales, la relación con otros sujetos y el acuerdo social.

La escuela primaria considerada es la única que existe en la localidad, y cuenta actualmente con algo más de 100 alumnos distribuidos de primero a sexto grado. Se halla inserta en un pequeño poblado, con algo menos de 1.000 habitantes, ubicado a 30 kilómetros de la ciudad de Villa María. Su principal fuente de recursos lo constituye la explotación ganadera, en lo que concierne a producción de leche y sus derivados.

La cercanía de una importante ciudad - como lo es Villa María - ha impedido el crecimiento demográfico de la población, la cual se mantuvo estable, en número de habitantes, con el correr de los años. Cabe aclarar que esta ciudad posee unos 90.000 habitantes, con una zona de influencia de aproximadamente 300.000 personas, distribuidas en localidades en un radio de $60 \mathrm{~km}$. Asimismo, es un verdadero nodo en el campo de las comunicaciones, ya que pasan por ella las principales rutas del país y posee impor- 
tantes centros de formación docente, tanto a nivel terciario como universitario, los que tienen gran influencia sobre las políticas educativas de la región.

\subsection{Justificación del estudio}

Consideramos que analizar el cuaderno y carpeta de clase adquiere relevancia en tanto resulta ser un ámbito de escrituración escolar que es guardado, coleccionado, revisado y revistado a lo largo del tiempo, dando cuenta de la "vida cotidiana de la escuela", en una época y en un lugar determinado. Asimismo, es un espacio de interacción entre docentes y alumnos, un lugar donde cotidianamente se enfrentan los actores del proceso de enseñanza y aprendizaje y, por tanto, es posible vislumbrar los efectos de la actividad escolar.

Otra importancia se encuentra en el hecho de que todos los días, en casi todas las horas de clase, alumnos y docentes llevan a cabo un minucioso proceso de escrituración, cuyos ámbitos de registro privilegiados son el cuaderno y el pizarrón, por lo que el primero se convierte en un campo significativo para observar los procesos históricos y pedagógicos de la enseñanza de la matemática acontecidos en la escuela.

\section{DISEÑO METODOLÓGICO.}

El estudio se enmarcó en una metodología cualitativa de investigación, en tanto se intentó arribar a una comprensión profunda de la actividad matemática desarrollada en los cuadernos y carpetas de clases a través de un análisis inductivo/constructivo (Lincoln y Guba, 1985), y no partimos de hipótesis previamente establecidas, sino que, a partir de los datos recogidos se generaron categorías y conjeturas cuya validez fue testada en el transcurso del trabajo. El recorte de nuestra investigación nos llevó a considerar sólo el sexto año de estudios, en tanto es el único curso, para 1955, con el que se tiene la documentación completa.

En la búsqueda de continuidades y discontinuidades que se presentaron en la enseñanza de la matemática, para estas tres generaciones, nuestra investigación nos llevó a considerar las siguientes dimensiones de análisis:

- Configuración de los cuadernos y carpetas de clase (ritualizaciones, uso de títulos, subtítulos, fechas, corrección de las actividades y tareas, etc).

- Contenidos matemáticos abordados y desarrollados.

- Jerarquía de los contenidos (subáreas de la matemática, interdisciplinariedad).

- La resolución de problemas (tipos de problemas y ejercicios tratados, cantidad de situaciones problemáticas presentadas, estilo de planteos que presentan los problemas, respuestas brindadas a los problemas y ejercicios, concepción del conocimiento matemático que se trasluce).

- Las evaluaciones administradas (tipos y cantidad de evaluaciones suministradas, relación entre evaluación y contenidos abordados, escala de calificación empleada).

Para efectuar el análisis de las mismas, decidimos transcribir las actividades de clase de los tres años lectivos, ya que facilitó el procesamiento de datos y resultó más eficiente una búsqueda en material digitalizado que en impresos, fundamentalmente porque los contenidos de matemática estaban intercalados con los de las demás disciplinas. 
Asimismo, la digitalización de la información nos permitió emplear coloraciones diferenciadas para las evaluaciones, contenidos conceptuales y procedimentales, áreas de la matemática abordadas y la utilización de marcadores para las actividades que presentaban relevancia para el estudio.

Es importante destacar que en el año 1993 comenzó un proceso de Transformación Curricular Federal en Argentina, acorde con lo previsto por la Ley Federal de Cultura y Educación. Dicha ley dispuso que el Consejo Federal de Cultura y Educación, presidido por el Ministro de Cultura y Educación de la Nación, aprobara los Contenidos Básicos Comunes (CBC) para todo el país. Hasta ese entonces, los procesos de cambio curricular se realizaban en forma heterogénea y no coordinados en los diferentes contextos provinciales.

Por esta razón, hemos considerado apropiado efectuar un análisis de los contenidos abordados en los tres años de estudio, poniéndolos a la luz de estos Contenidos Básicos Comunes, en tanto se tiene un parámetro de comparación, y fundamentalmente, porque consideramos que los mismos presuponen una reflexión crítica al estado actual, remitidos a ese momento, de la enseñanza y aprendizaje de la matemática.

\section{RESULTADOS Y DISCUSIÓN}

\subsection{Configuración de los cuadernos y carpetas de clase}

Describiremos de manera general - y tal vez obvia, aunque no por ello menos importante - la estructura de los cuadernos y carpetas de clase de matemática que se utilizaron durante los años 1955, 1978 y 2002, correspondientes al sexto año de estudio, dentro del subsistema de enseñanza primaria de Argentina, de la escuela considerada.

Cabe destacar que el cuaderno de clase usado en nuestro país fue único hasta la década del setenta, en el que toda ejercitación y actividad, sin importar de qué materia fuese, se encontró incluida en el mismo. A partir de allí, se utilizaron hojas individuales, generalmente de tres perforaciones y unidas con argollas metálicas, y se separó el trabajo áulico en dos carpetas; por un lado matemática y ciencias naturales, y por el otro, lengua y ciencias sociales.

Tanto los cuadernos como las carpetas se estructuraron en función de tres ejes centrales que se presentaron subordinados entre sí. Para 1955 y 1978 estos ejes lo constituyen el tiempo, el área de la matemática a ser abordada y el contenido disciplinar a tratar. Para el año 2002 los ejes fueron el tiempo, el tema de matemática a ser abordado y la actividad de la clase.

El tiempo, dividido en meses y días, es el primer eje a partir del cual los cuadernos y las carpetas se ordenaron, y cada día que se inició fue taxativamente indicado. Ninguna fecha se salteó a menos que se tratara de un fin de semana, un feriado o recesos escolares. Si existió actividad escolar, inequívocamente se puede constatar el día en el que fue realizada; es decir, si hubo trabajo, existe el registro de la fecha de realización. Una nueva fecha siempre coincidió con el inicio de una nueva actividad, y por lo tanto, cada día tuvo una fuerte correspondencia con un número determinado de actividades realizadas.

Los cuadernos de clase de 1955 presentan, además, una doble línea ubicada en el centro del renglón para marcar el final de una actividad, de una jornada de trabajo o de un día, y el comienzo del otro. A continuación se indicaba fecha, rama de la matemática y tema a ser abordado. Por su parte, en el año 1978 las actividades de cada día también fueron separadas por una doble línea que atravesaba toda la hoja, y los 
contenidos matemáticos se alternaron con los de ciencias naturales. A continuación, también se indicaba la fecha, área en la que los alumnos se encontraban trabajando y finalmente el tema a ser abordado.

Para el año 2002 se continuó con el ritual de indicar la fecha al comienzo del día, luego el tema y la actividad a ser desarrollada. Los contenidos también se encontraron alternados con los de ciencias naturales.

Para los tres años estudiados, las actividades de matemática se sucedieron al iniciar la jornada escolar, razón por la que consideramos fue el motivo para que se incluyera la fecha, puesto que no es observable la misma en las demás disciplinas que se abordaron a lo largo del día.

Por otra parte, cabe destacar que los cuadernos de 1955 presentan una escritura monocromática en tinta negra o eventualmente azul marino, y la utilización del color se limitó sólo a las construcciones geométricas. En 1978, la escritura en la carpeta de matemática se realizó en color azul y la utilización de una gama restricta de colores - que incluyen el verde, rojo y negro - para el subrayado de títulos, esquemas e ilustraciones.

El año 2002, la carpeta de matemática presentó una mayor diversidad en coloración para la escritura, puesto que se utilizó de manera aleatoria diferentes colores, ya sea para el subrayado de títulos y subtítulos, distinguir ejercicios, respuestas a ejercicios y problemas, propiedades, etc.

Sólo en los cuadernos de clase de 1955 se puede constatar la existencia de actividades destinadas a que los alumnos las efectuaran en el hogar. Las mismas están tituladas con "Deber" o "Deberes" y sobre un total de 104 clases (descontando instancias de evaluación) 28 las presentan (27\%). En este sentido, como señala Gvirtz (1997), el tiempo en la escuela es un constructo que implica necesariamente la finalización de las tareas, y el día no se termina en tanto no se termina la tarea o actividad estipulada. Asimismo, si una tarea no fue realizada, tiende a ocupar en el cuaderno el lugar y tiempo correspondiente al ritmo social que le impone la escuela, y se denuncia la misma con un "Incompleto" o "Completar".

El tipo de corrección que se presenta en los cuadernos de 1955 se condice con las pautas impuestas por el sistema escolar de la época, por las cuales se exigió a los maestros corregir al alumno en el momento preciso en que se cometía un error. El control de la escuela no se hallaba solamente sobre las personas sino sobre el trabajo de los mismos, fundamentalmente lo enseñado por el docente. Asimismo, la corrección debía hacerse en rojo, en tanto el color era asociado por los pedagogos de la época con el lema "la letra con sangre entra" (Gvittz 1997, p.56 - 57). En consecuencia, todas las actividades de clase de 1955 están corregidas exclusivamente por la maestra y las mismas presentan una grafía en representación de un "bien" o "mal", o eventualmente alguna nota que incita al alumno a que mejore la caligrafía u ortografía.

En 1978 la actividad de corrección continuó centrada prioritariamente en el docente, aunque se utilizó el color verde con exclusividad. Fortuitamente se presentan algunos ejercicios visados por el alumno y luego por la maestra. A diferencia de las correcciones de 1955, las actividades fueron valoradas con expresiones tales como: "Excelente", "Muy Bueno", "Bueno", "Satisfactorio" o "No satisfactorio".

En el año 2002 se comparte la tarea de corrección, puesto que la mayoría de los ejercicios y actividades de clase están visados por el alumno y luego presentan una apreciación general del docente, con valoraciones similares a las utilizadas en 1978. 
Al visado de las actividades por parte del docente, debe anexársele la corrección por parte del director de la escuela, aunque la misma es debida, como lo expresa Gvirtz (1997, p. 53 - 54), a mecanismos de control impuestos sobre el trabajo del maestro y no del alumno. Estos visados sólo fueron encontrados en los cuadernos de 1955 y en la carpeta de clase de 1978.

Finalmente, las efemérides dan testimonio dentro de los cuadernos y carpetas de clases de matemática. Si bien en nuestro país las fechas más festejadas aluden al 25 de mayo de 1810; 20 de junio de 1820; 9 de julio de 1816; 17 de agosto de 1850, 11 de septiembre de 1888 y 12 de octubre de 1492; también hallamos otros acontecimientos, como por ejemplo: Día del Animal, Día del Ejército Argentino, entre otros.

\subsection{Los contenidos matemáticos abordados.}

Consideramos a continuación los contenidos explicitados en los cuadernos y carpetas de clase y que han sido abordados durante los años lectivos 1955, 1978 y 2002. Los mismos han sido reorganizados en bloques con la finalidad de facilitar el análisis y la lectura. No obstante, cabe aclarar que no fueron tratados en las clases de esa manera y la secuencia que exponemos dista mucho de lo que en realidad sucedió.

Llama la atención que los diferentes contenidos se presentaron entremezclados, sin conexión alguna entre sí, y se alternaron las clases de geometría con las de aritmética; o aritmética, geometría, álgebra y estadística para el año 2002. Sólo para el año 1955 se advierte que los contenidos relativos a mediciones atravesaron todas las actividades desarrolladas en el año y se presentaron en la mayoría de las situaciones problemáticas o actividades de clase, y no existe un contenido común que atraviese los años 1978 y 2002.

Los contenidos relativos a números naturales fueron abordados en los tres años de estudio, aunque no consideramos que el enfoque que tuvo haya dejado claro en los alumnos el uso que tienen los números fraccionarios y los decimales, como así también, la equivalencia entre ellos, puesto que no encontramos registros que lo marcaran taxativamente. En este sentido, notamos que la presentación de los diferentes campos numéricos se efectuó de manera abstracta y su uso deviene de las inferencias que pudieran realizar los alumnos a través de las situaciones problemáticas que se le presentaron como ejercitación, aunque las mismas no agotaron el amplio espectro de aplicación que tienen estos números.

En este sentido, encontramos sólo una actividad - desarrollada en el año 2002 - que presenta la utilidad que podrían tener los diferentes números, aunque en un contexto particular, como son los precios de los productos en un mercado.

Esta separación en compartimentos de los contenidos también se presentó con "porcentajes", los cuales estuvieron asociados al cálculo de interés en 1955 y como un tema en sí mismo para el año 1978, aislado de números decimales y racionales, en contradicción con lo que se recomienda en los CBC. No obstante, hallamos dos actividades prácticas en el 2002 que integran porcentajes con números racionales, las cuales, debido al enfoque particular que se le dio, no nos da seguridad de que los alumnos se hayan percatado de su importancia.

En el caso de las fracciones, es de destacar que fueron presentadas como partes de un todo discreto, sin aprovechar los múltiples significados que se le pueden dar a las mismas (como medida, relación entre partes, porcentaje, razón, probabilidad de que ocurra un suceso, entre otras). Tampoco encontramos que se presentaran las relaciones que existen entre los números racionales y los naturales (u otros campos nu- 
méricos), lo que brinda la posibilidad de conectar lo nuevo con lo ya conocido y hace que los alumnos encaren el aprendizaje de una forma reflexiva.

Con respecto al orden entre fraccionarios, percibimos que en el año 2002 se establecieron reglas de comparación teniendo en cuenta los numeradores y los denominadores; análisis que a nuestro juicio suele provocar confusión en los alumnos, que dudan respecto a qué parte le corresponde el signo $\rangle,\langle 0=$, o consideran que una fracción está compuesta por dos partes, de forma tal que cada una de ellas pueda ser tomada como un número entero con la posibilidad de ser comparado con su correspondiente en otra fracción.

Los números enteros sólo fueron trabajados de manera general en 1978 y abordados con mayor profundidad en el 2002, aunque limitando el tratamiento a las operaciones de suma y resta. En este punto, es de destacar que su enseñanza no ha sido estipulada por los CBC.

Con respecto a las operaciones con números, percibimos que en los tres años se presentaron los algoritmos de suma, resta, multiplicación y división de racionales, apelando a reglas instituidas y prescindiendo de la comprensión de los mismos por parte de los alumnos. Los algoritmos fueron presentados en primera instancia y posteriormente se realizaron gran cantidad de ejercicios con la finalidad de fijar el procedimiento, ignorando la equivalencia entre fracciones que había sido abordada cuando se introdujo el tema.

Hacemos notar que los diferentes lineamientos curriculares para la enseñanza primaria, marcan insistentemente que los algoritmos no son el punto de partida de una operación, y que su construcción implica el descubrimiento de las relaciones puestas en juego y el análisis de las situaciones en las cuales se puede aplicar, y a pesar de ello, su enseñanza va en contra de estas recomendaciones metodológicas. Al respecto, consideramos que comprender una operación entre números implica atender a los conceptos y relaciones que se presentan en los procedimientos, y no sólo a las formas o técnicas de cálculo de sus resultados, las que atentan contra una genuina comprensión.

No se trabajó con ecuaciones en los años 1955 y 1978 y sí en el año 2002, aunque de manera abstracta y con pocas vinculaciones con el mundo real y la resolución de problemas. Asimismo, la resolución de ecuaciones aconteció después de establecerse algunas reglas de transposición de términos, con una considerable cantidad de ejercicios que aparentemente tenían la finalidad de fijar algoritmos.

A su vez, resulta visible en diversas actividades relacionadas con ecuaciones, una incorrecta aplicación de la transposición de términos para la división y multiplicación, la que pasa inadvertida, tanto para la docente como para los alumnos, por no efectuarse la verificación del valor numérico hallado para la variable.

También hallamos, para el año 2002, una inadecuada aplicación de las ecuaciones a situaciones concretas, las cuales no fueron identificadas por el alumno como susceptibles a ser manipuladas desde ese enfoque. En este sentido, hacemos notar que las ecuaciones no fueron presentadas como una herramienta que permite resolver situaciones problemáticas, sino más bien, un camino para expresar en lenguaje algebraico algunas situaciones que pueden prescindir de ellas.

Las desigualdades sólo fueron abordadas en los años 1955, 1978 y 2002 como instancias de comparación y ordenación entre números, y no como inecuaciones. Tampoco se trabajó en estos años con el cálculo aproximado y redondeo de cantidades.

A pesar que "proporcionalidad" es un tema prescripto en los CBC, no fue planteado en el año 2002. El tratamiento de esta temática la encontramos en 1978 y abordada con mayor profundidad en 1955, año en 
el cual no sólo se trabajó con proporcionalidad directa e inversa, sino también con proporcionalidad compuesta, directa e inversa, y cálculo de interés. La ausencia del tratamiento de proporcionalidad, durante el año lectivo 2002, se pone en evidencia al realizarse conversiones de unidades del sistema sexagesimal de manera incorrecta, por ignorarse el factor de proporcionalidad que estaba involucrado.

Las nociones geométricas se trabajaron en los tres años objeto de estudio, aunque se presentan abordadas con mayor profundidad en el año 1955, con un recorte de contenidos en 1978 y de manera sucinta en el 2002. No obstante, se advierte un denodado esfuerzo en el año 1955 por relacionar la geometría con problemas que pudieran presentarse en el entorno cotidiano del alumno, aunque muchos de ellos pueden caer en la categoría de ficticios o poco relevantes.

El abordaje que presentó "recta y plano", para los tres años se estudio, consistió en la realización de una presentación geométrica de dichos conceptos, con escasas relaciones con el entorno inmediato del alumno. Las construcciones con regla y compás presentan un tratamiento minucioso en el año 1955, en el que se inscriben hasta pentágonos regulares en una circunferencia, aunque se excluyen los fundamentos de la construcción.

De todos modos, las construcciones geométricas, presentadas en los tres años, se llevaron a cabo como un conjunto de reglas de acción y secuencias que constituyen la técnica de construcción, desechándose por completo los principios geométricos inmersos. Así, la construcción de un triángulo equilátero deviene de efectuar "dos arcos" con el compás después de tomar como centros los extremos de un segmento, sin tener en cuenta que la congruencia de los lados está garantizada por las dos circunferencias que tienen por radio el segmento dado.

Cuerpos geométricos fue abordado en 1955 y 1978 con cálculos de superficies laterales y volúmenes, en tanto que en el año 2002 sólo se presentó una clasificación de los mismos, nombrándose algunos elementos representativos. Finalmente, si bien los CBC establecen la obligatoriedad de la enseñanza de movimientos en el plano, estos temas no fueron abordados en el año 2002, aunque tampoco se presentaron en 1955 y 1978.

Todo lo relativo a mediciones se abordó con mayor profundidad, tanto desde el punto de vista conceptual como procedimental, durante el año 1955, en menor grado en el año 1978 y de manera muy escueta y superficial en el año 2002 (área y perímetro de un triángulo y amplitud de un ángulo). Notamos, además, que la presentación de las fórmulas relativas a perímetros, superficies de figuras y cuerpos, ha sido establecida por el docente, en los tres años objeto de estudio, desechándose las instancias de construcción de las mismas y de exploración acerca de su origen y esencia. En este sentido, pensamos que la enseñanza de la geometría debería contribuir a que los alumnos comprendan qué son y para qué sirven los contenidos, dejando de lado un aprendizaje imitativo y memorístico, basado en el ver, nombrar y definir.

Asimismo, observamos que las situaciones problemáticas de geometría, que se expusieron en los tres años lectivos estudiados, implican por lo general la aplicación de una o varias fórmulas asociadas al cálculo de superficie libre o remanente en figuras compuestas.

Por otro lado, vemos que el año 2002 presenta una ausencia total del tema "sistema de unidades", el cual es básico para otros campos disciplinares, como biología, física, astronomía, geología, etc. A pesar de que en 1955 y 1978 se abordó con suficiente profundidad este tópico, no se trabajó con los errores que se producen en las mediciones y cálculo de medidas. 
Con respecto a los contenidos relativos a estadística y probabilidad, hallamos que las nociones elementales sólo fueron trabajadas durante una clase del año 2002, a través de la lectura de 3 gráficas de un material impreso que se les entregó a los alumnos. Esta actividad no fue evaluada por la docente y no formó parte de instancias de evaluación.

\subsection{Jerarquización de los contenidos}

La utilización del término "jerarquización” obedece a que los saberes se pueden ordenar según una frecuencia de aparición en los cuadernos. De esta manera se transforman las diferencias cualitativas de los saberes en cuantitativas y, en función de ello, se establece la posibilidad de jerarquizarlos.

En este sentido, la jerarquización de contenidos que hemos realizado apunta a cuantificar los saberes por área de la matemática a la que pertenecen. Es notable que en los cuadernos de 1955 la separación que se encuentra es: "Aritmética" y "Geometría", tal como era realizada en la antigüedad por los griegos, quienes distinguían como objeto de conocimiento, por un lado, el estudio de las propiedades de los números y las operaciones, $y$, por el otro, el estudio de las formas de los cuerpos y las relaciones entre sus elementos.

En la carpeta de 1978 la separación también se establece por área y así se tiene: "Área Matemática" y "Área Geometría". No obstante, si consideramos los contenidos abordados en la primera, notamos que se corresponden con aritmética. Para el años 2002 desaparece la separación por áreas de la matemática y sólo se alude a los temas que son abordados.

Para los años 1978 y 2002, los temas desarrollados se encuentran enmarcados en unidades didácticas cuyos títulos no guardan relación con los contenidos abordados, y la denominación empleada alude a temas de las ciencias naturales. Tampoco hallamos situaciones problemáticas que vincularan los temas de matemática con los de ciencias naturales, por lo que el nombre de la unidad resultó totalmente ficticio e inapropiado para los contenidos matemáticos abordados.

Analizando la cantidad de clases que se destinaron a cada área de la matemática durante los tres años objetos de estudio, se evidencia el paulatino abandono que tuvo la geometría en el currículo escolar, tal como es denunciado desde hace tiempo por distintos investigadores de Educación Matemática, y la permanencia de la aritmética. Asimismo, aparecen hacia el 2002 otras ramas de la matemática que se condicen con las nuevas reformas educativas y con los avances del conocimiento científico en esta disciplina, tal como lo es álgebra y estadística.

\section{$3.4 \quad$ La resolución de problemas}

En las consideraciones metodológicas de los $\mathrm{CBC}$ encontramos que se hace un fuerte hincapié en algunos métodos didácticos, calificados de trascendencia para la enseñanza de la matemática, y por lo tanto, recomendados metodológicamente. Entre ellos se tiene la resolución de problemas y la modelización.

La incorporación de la resolución de problemas como estrategia metodológica, en los CBC, sienta sus bases en el hecho de involucrar procesos básicos del pensamiento matemático en los alumnos, fundamentalmente los que devienen de las cuatro fases que distingue Polya (1957) en relación a los procedimientos heurísticos, algorítmicos y pensamiento divergente aplicados en la resolución de situaciones problemáticas. 
La modelización, por su parte, se fundamenta en el hecho de que el alumno relaciona los conceptos matemáticos con la realidad y logra entender la necesidad del estudio de esta disciplina y su importancia en la aplicación a otras.

En cuanto a la resolución de problemas, cabe aclarar que hay una diferencia básica entre el concepto "problema" y "ejercicio", por lo que no es lo mismo hacer un ejercicio que resolver un problema. Una cosa es aplicar un algoritmo de forma más o menos mecánica, evitando las dificultades que introduce la aplicación de reglas cada vez más complejas, y otra, resolver un problema, lo que conlleva dar una explicación coherente a un conjunto de datos relacionados dentro del contexto.

Como expresa Guzmán (1992), se tiene un verdadero problema cuando uno se encuentra en una situación desde la que se quiere llegar a otra, unas veces bien conocida, otras un tanto confusamente perfilada, y no se conoce el camino que puede llevar de una a otra.

Al respecto, Pólya (1957) clasifica los problemas en cuatro categorías: Problemas operativos o de rutina (considerados éstos esencialmente como ejercicios de aplicación); Problemas por resolver (problemas elementales no triviales); Problemas por demostrar y Problemas abiertos. De acuerdo a la concepción de problema expresada anteriormente, estas categorías resultarían ser sólo tres, ya que "los problemas operativos" son meros ejercicios y no verdaderos problemas. No obstante, bajo estos criterios hemos tipificado y cuantificado las situaciones problemáticas que se les presentaron a los alumnos durante los años 1955, 1978 y 2002.

Al respecto cabe aclarar que no encontramos problemas por demostrar o problemas abiertos durante los años objeto de estudio. Sólo hallamos problemas por resolver, los que fueron incorporados en esta categoría bajo un criterio amplio de aceptación, puesto que de lo contrario, un análisis minucioso nos hubiese llevado a catalogarlos como problemas operativos. Hemos considerado problemas por resolver a aquellas situaciones problemáticas que excedían un simple cálculo o aplicación de fórmula, demandando por parte del alumno un análisis del problema, el establecer relaciones, aplicar estrategias, recursos o métodos para solucionarlo.

Con respecto a los "problemas operativos", hemos contabilizado cada una de las situaciones que se le presentaron al alumno y que no tuvieran nexos entre sí. De esta manera, si el ejercicio solicitaba hallar los divisores de 6 números enteros, registramos 6 situaciones a resolver. Si se presentaba una tabla de cálculo de operaciones con $n$ por $m$ entradas, consideramos que existían (n.m) situaciones a resolver por el estudiante.

El análisis de los datos obtenidos muestra una tendencia, para los años 1955 y 1978, a trabajar los problemas operativos en la primera mitad del año lectivo, en tanto para el año 2002, estos problemas se presentan con mayor frecuencia hacia la segunda mitad del año.

Con respecto a los "problemas por resolver", se halla una mayor frecuencia de los mismos cuando se superó la mitad del año lectivo, para los tres años objeto de estudio. De todos modos, considerando el total de problemas trabajados por año tenemos: 


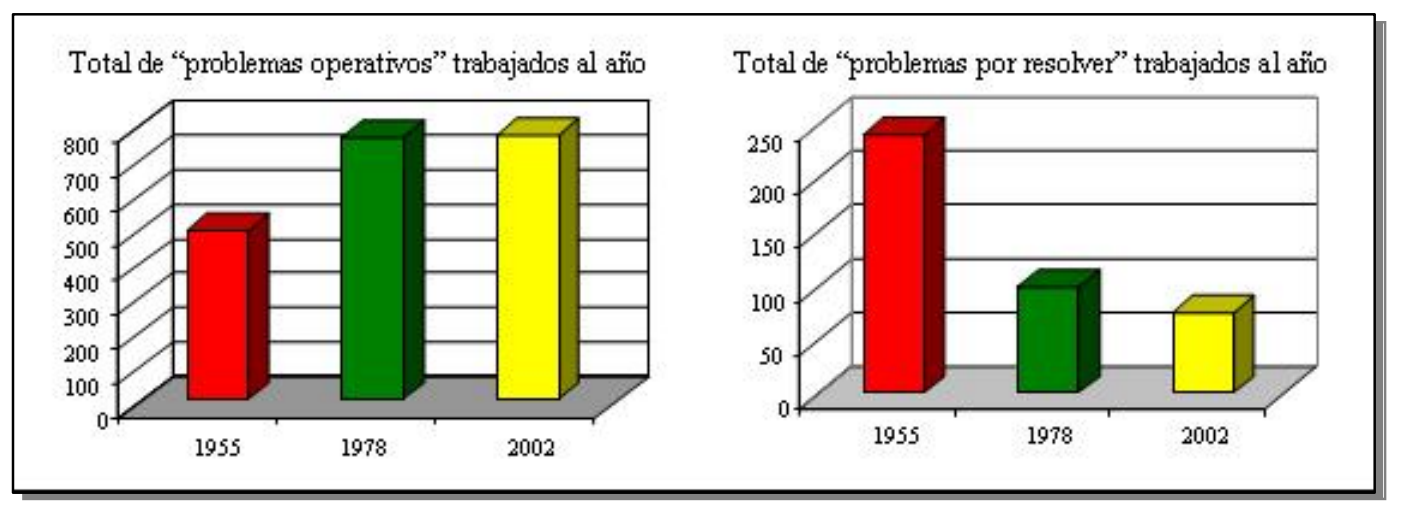

Es apreciable, que durante los años 1978 y 2002, la resolución de problemas estuvo centrada en la "resolución de ejercicios", en tanto que 1955 se lo puede caracterizar por trabajar considerablemente los "problemas por resolver". Asimismo, también es notable la disminución que se tuvo en la cantidad de problemas que se trabajaron con el correr de los años, puesto que de 240 que se presentaron en 1955, llegamos a un total de 75 para el 2002, siendo que estuvo prescripto y recomendado desde los CBC desde 1993.

La insistencia que se presenta, entre las recomendaciones metodológicas de los CBC para que se trabaje con problemas que adquieran significado para los alumnos, y que se hallen relacionados con su entorno inmediato, nos llevó a discriminar entre los "problemas por resolver" aquellos que pertenecen a un contexto real y de la vida cotidiana, de los que se encuentran en un plano abstracto y netamente matemático.

Discriminando las situaciones problemáticas trabajadas en los años 1955, 1978 y 2002 en: "Problemas en contexto real" y "Problemas en contexto abstracto" encontramos una tendencia a efectuar mayor cantidad de problemas operativos al transcurrir los años, y por ende, una disminución de los que hemos llamado "problemas por resolver". Asimismo, entre las situaciones que presentan mayor interés, desde el punto de vista didáctico, se muestra una propensión a trabajar con problemas ajenos al mundo real y contexto cotidiano de los alumnos. En este punto, cabe aclarar que acordamos en que existen problemas sumamente motivadores que escapan de la realidad y espacio temporal actual, y que catalogamos como apropiados para trabajar con los alumnos; pero no fue este el caso entre los que analizamos, puesto que sólo hallamos situaciones que relacionaron áreas entre figuras geométricas, matizadas con algunas operaciones algebraicas, tal como aparecen en muchos de los textos escolares y que catalogaríamos de triviales.

Debido a que nuestro análisis se centró en los cuadernos y carpetas de clase, no es posible determinar a ciencia cierta si hubo un trabajo a conciencia con la "diferenciación de las situaciones problemáticas" tal como se establecen en los CBC. Sólo hallamos en los cuadernos de 1955 un esfuerzo por pautar algunas etapas de la resolución de problemas, en el sentido que cada situación presenta un cuidadoso planteo (aunque se omiten las operaciones algebraicas), una respuesta y una explicación del "razonamiento" seguido para resolver el problema. No obstante, creemos que exigir la solución de una situación problemática de una manera determinada, empobrece la búsqueda e interfiere la autoconstrucción de los conocimientos.

En los restantes años, o sea 1978 y 2002, los problemas presentan el desarrollo algebraico necesario para resolverlo y una respuesta que no es sólo numérica, sino mas bien, reproduce palabras expresadas en la interrogación de la situación. 
Finalmente, subrayamos que en los tres años objeto de estudio no hallamos instancias de una verdadera modelización o de creación de problemas, en tanto los alumnos se limitaron a resolver las situaciones planteadas por el docente.

\subsection{Las evaluaciones administradas.}

A través de la evaluación se otorga un valor al trabajo realizado. El mismo irá a depender de la distancia entre lo efectuado y lo solicitado. La evaluación llevada a cabo en los cuadernos y en las carpetas podría subdividirse, en este sentido, en dos acciones bien diferenciadas. Por un lado se presenta la calificación, en donde a la actividad desarrollada se le otorga una nota de acuerdo a la distancia en que se encuentra respecto del punto de llegada; y por otro lado, una segunda actividad: la corrección, que consiste en señalar los errores e indicar lo que hubiera sido esperable. La corrección explicita además los parámetros utilizados al calificar, con lo cual legitima la misma.

Sabemos que el valor dado en las calificaciones es susceptible de ser medido en alguna escala, ya sea nominal o numérica. Así, pudimos constatar que la medición ha sido numérica para 1955, tal como se utiliza en estos momentos para el Nivel Medio y Superior de la Educación Argentina. Para los años 1978 y 2002 se utiliza un adjetivo calificativo, acompañado por una cuantificación en porcentaje alcanzado. A su vez, todas las evaluaciones administradas en 1955 y 1978 presentan la firma del padre o madre del alumno, no ocurriendo de esta manera con las correspondientes al año 2002.

Considerando los tres años, vemos que difieren en la cantidad de evaluaciones administradas (16 evaluaciones en el año 1955 y 8 para los años 1978 y 2002). No sólo la diferencia se encuentra en cantidad, sino también, en extensión y forma en que fueron administradas, puesto que en 1955 las evaluaciones se llegaron a tomar hasta en cuatro días consecutivos, fraccionando los temas, y abarcaron todos los tópicos tratados.

Tanto para 1955 como para 1978, los contenidos evaluados guardan relación con los trabajados en clase; y la mayoría de las situaciones problemáticas presentadas difieren en cantidades numéricas con respecto a las realizadas. Además, sólo en 1955 se evaluaron todos los temas trabajados durante el año lectivo, en tanto que para 1978 y 2002, quedaron sin evaluar los contenidos desarrollados en el último mes de clases y una gran parte de los abordados en geometría.

No todas las actividades que presentan las evaluaciones suministradas en el 2002 guardaron relación con las trabajadas en clase. Hallamos, en algunos casos, situaciones que relacionaron contenidos matemáticos con situaciones del mundo cotidiano, a pesar de no haber sido enfocada de esa manera la enseñanza de los contenidos que las involucraban. Si bien este enfoque condice con los lineamientos curriculares para el nivel primario, ofrecidos por la Secretaría Ministerio de Educación (1987) - en la sección de instrumentos de evaluación ${ }^{1}$ - no compartimos el criterio de evaluar a los alumnos sometiéndolos a la resolución de problemas que poco y nada tienen que ver con lo abordado en clase, solicitando que hagan conexiones entre contenidos y extrapolen conocimientos a otras áreas, cuando no fue esa la acción didáctica promulgada en las clases. No estamos diciendo con esto que concebimos a la evaluación como la reproducción de situaciones que ya se trabajaron y que se deben presentar levemente modificadas en sus valo-

\footnotetext{
1 "Para valorar el rendimiento, se dará importancia a la necesidad de comprobar la integración de aprendizaje y la confrontación del alumno con situaciones inéditas, realzando las condiciones en que el alumno resuelva situaciones problemáticas con los elementos informativos a su alcance". SME (1997, p. 27).
} 
res numéricos; sino más bien, no adherimos en que la misma se convierta en un instrumento que mida capacidades y/o habilidades que no se enseñan o que no están presentes en la metodología de trabajo del docente.

\section{CONCLUSIONES}

Llegamos al momento del cierre de nuestra investigación, la cual tuvo por objetivo analizar las continuidades y discontinuidades que tuvo la enseñanza de la matemática en tres generaciones, que cursaron el sexto año de estudio, en una misma escuela primaria.

Entre las continuidades que se mantienen para los tres años lectivos analizados $(1955,1978$ y 2002) hallamos que:

- Se continúa con el ritual de ordenar las actividades de cada día colocando una fecha, la que marca un tiempo escolar en el que se encuadra el trabajo áulico.

- Se mantienen en la currícula gran parte de los contenidos matemáticos que fueron recomendados desde los CBC para que el alumno adquiera gradualmente los procesos de pensamiento propios de la matemática.

- El conocimiento matemático se halla fragmentado, en tanto los contenidos han sido divididos en temas y subtemas, y su presentación se aborda sin la existencia de nexos entre sí, por lo que cada ejercicio y situación problemática tratada se corresponde sólo con un tópico y poco tiene que ver con los restantes. Este proceso es observable, en primera instancia, a través de los títulos y/o subtítulos que indican la referencia temática, y en el estilo que ha conservado la escuela al presentar de manera alternada los contenidos de aritmética, álgebra y geometría, excluyendo los vínculos que existen entre ellos. Así, ha logrado que el saber se suministre a los alumnos en "cómodas cuotas" y la totalidad del mismo se obtenga por yuxtaposición de partes con escasas interrelaciones entre sí.

- No existe un trabajo interdisciplinario entre la matemática y las restantes disciplinas. La integración de matemática y ciencias naturales, para 1978 y 2002, fue aparente y carente de autenticidad, en el sentido que se hallaron unidas en una misma carpeta sin interconexiones entre ellas.

- Se ha dado prioridad a la memorización de conceptos y técnicas, sin preocupación por la comprensión de las estructuras que las justifican; lo que fomentaría una visión de tipo mecánico de la disciplina, en donde el estudiante puede considerar que lo esencial es utilizar de manera sistemática una serie de procedimientos algorítmicos ejecutados con cierta rapidez.

- La mayoría de las consignas de las actividades se construyen con un solo verbo o una simple proposición, tales como: "Reducir", “Completa”, "Hallar el...", "Simplificar", "Escribe cómo se leen", etc. De esta manera, las tareas constituyen más órdenes que consignas y el resultado es una respuesta única. Ninguna de las tareas se presenta enmarcada en un contexto social que pueda emular situaciones de la vida cotidiana, como presentar un presupuesto, calcular los materiales de una obra o modelizar una situación real. 
- Se fomenta el aprendizaje repetitivo, en donde la mayoría de los ejercicios, por su carácter rutinario, activan procesos de pensamiento muy circunscrito a la lógica que opera en el aula. El pensamiento creativo pierde importancia al no existir un espacio en el que sea promovido, puesto que no hallamos la realización de tareas optativas, las que podrían activar un pensamiento divergente o lateral a partir de los intereses, motivaciones y niveles de aspiración de los propios alumnos.

Como discontinuidades en la enseñanza de la matemática, para los años lectivos 1955, 1978 y 2002, hallamos que:

- La carpeta de clase se volvió heterogénea, en el sentido que el alumno tiene libertad de escribirla a su manera, aunque continuó la homogeneidad en el conocimiento impartido.

- Las evaluaciones perdieron la rigurosidad que tenían en el año 1955, en el cual se evaluaban minuciosamente todos los conocimientos a través de pruebas exhaustivas y extensas, las que demandaban más de un día para ser administradas.

- La corrección de las tareas escolares dejó de ser una tarea prioritaria del docente y pasó a ser compartida con los alumnos, desapareciendo, asimismo, el control del personal directivo sobre la tarea escolar.

- Desaparecieron las efemérides en la carpeta de matemática y el trabajo de clase se convirtió en "rellenar" espacios vacíos en copias impresas. Por consiguiente el alumno escribe menos en matemática en comparación con el año 1955. Asimismo, la grafía de los cuadernos del año 1955 presentan a primera vista, un trazo homogéneo y parecen estar realizadas por un adulto y no por un niño.

- Se eliminaron los "deberes" hogareños, pasándose a desarrollar gran parte de las actividades en horas de clase, y relegando para el hogar aquellas tareas que no llegó a terminar el alumno.

- Las respuestas a las situaciones problemáticas no tienen patrones únicos que permitan una categorización como en 1955. En los años 1978 y 2002 pueden ocupar más o menos espacio y ser más o menos precisas, sin embargo, están directamente relacionadas con el tipo de actividad y reproducen expresiones contenidas en el problema.

- Se califican las actividades de clase asignándosele una valoración por parte del docente, tal como "Excelente", "Bueno", etc. y las palabras "bien" o "mal", utilizadas en las correcciones de 1955, son utilizadas por el alumno para realizar la autocorrección de sus tareas.

- Desaparecieron contenidos de geometría plana y del espacio, confinando su enseñanza a la adquisición de definiciones, descripciones de cuerpos y figuras, y la aplicación de fórmulas en problemas sencillos, circunscriptos al cálculo de perímetros o superficies.

- Se abandona la partición de la matemática en "aritmética" y "geometría", apareciendo nuevas áreas, como álgebra y estadística, y nuevos temas, como sistemas de numeración en bases distintas a diez y números enteros.

- Desaparecieron contenidos conceptuales de vital importancia para el desempeño del alumno en la vida diaria $-\mathrm{y}$ vinculados fundamentalmente con otras áreas del conocimiento - donde la 
matemática resulta una herramienta útil. Entre ellos se encuentra proporcionalidad y unidades de medidas.

- Se realiza una mayor cantidad de ejercicios algorítmicos y se disminuye considerablemente la cantidad de problemas, especialmente de aquellos que pueden motivar e incentivar a pensar a los alumnos.

- Se utilizan frases que animan al trabajo en clase, tales como "Para que resuelvas con lo que sabes!”, "No olvides separar en términos!”, "iPara pensar y resolver!”, etc., sumadas a consignas que se asemejan al lenguaje coloquial que seguramente mantiene el docente con los alumnos.

- Las actividades presentan gráficos, ilustraciones y esquemas, propiciados básicamente por el desarrollo de la tecnología del fotocopiado.

- Aparece una mayor cantidad de errores conceptuales - en el desarrollo de los contenidos - por parte del docente a cargo de la asignatura.

Como cierre de nuestro trabajo, nos restan algunas reflexiones, que si bien no somos los primeros en decirlas, consideramos de gran importancia rescatarlas. La primera de ellas se halla relacionada con la resolución de problemas, cuyo abordaje, como lo advertimos, se ha limitado en los últimos años a la realización de meros ejercicios y no verdaderos problemas, lo cual es un gravísimo error. Sobre estas cuestiones, el mismo Pólya (1957) expresaba: Limitar la enseñanza de las matemáticas a la ejecución mecánica de operaciones rutinarias es rebajarlas por debajo del nivel de un "libro de cocina", ya que las recetas culinarias reservan una parte a la imaginación y al juicio del cocinero, mientras que las recetas matemáticas no permiten tal cosa.

Por otro lado, es común que los educadores le adjudiquen una parte importante de los errores, en la resolución de problemas, a las dificultades de comprensión lectora que presentan los alumnos. La tendencia a operar todos los datos presentados, vengan o no a consideración, certifica esta falta de comprensión global. Además, es por todos aceptado que los alumnos resuelven mejor los problemas si alguien se los lee que si lo hacen ellos mismos; lo cual constituye un error pedagógico muy frecuente, porque cuanto más facilitemos los docentes el aprendizaje, menor será el esfuerzo del alumno por aprender y por tanto, menor será la construcción de conocimientos. Asimismo, es notorio que las consignas y actividades que se les presentan a los estudiantes, tienden a ser cada vez más escuetas y precisas, e inducen a una menor lectura, simplificando al mismo tiempo las situaciones que se les proponen.

Finalmente, nos resta revalorizar la función que tienen los cuadernos y carpetas de clases, tal como lo propone Gvirtz (1997, p. 121), en tanto no son un mero soporte físico, por el contrario, se convierten en un dispositivo cuya articulación genera efectos, puesto que estructuran la dinámica del aula y permiten analizarla. Del mismo modo, retomamos un interrogante que se desglosa cuando comenzamos con nuestro trabajo: ¿Todo tiempo pasado fue mejor? No lo sabemos y no hay por qué tomar partido desde la investigación. Son los docentes, padres, alumnos, y todos los actores de la comunidad educativa, a quienes en cada contexto les corresponde la decisión. 


\section{BIBLIOGRAFÍA}

GuZMÁN, Miguel (1992). Tendencias Innovadoras en Educación Matemática. Buenos Aires: OMA.

GviRTZ, Silvina ((1997). Del currículo prescripto al currículo enseñado: Una mirada a los cuadernos de clase. Buenos Aires: Aique.

LINCOLN, Yvonna y GUBA, Egon. (1985) Naturalistic Inquiry. SAGE Publication.

Ministerio de Cultura y EduCAcion de la Nación (1995). Contenidos Básicos Comunes para la Educación General Básica. Buenos Aires: MCyE.

MinISTERIO de CULTURA Y EdUCACION DE LA NACIÓN (1996). Fuentes para la transformación curricular - Matemática. Buenos Aires: MCyE.

Ministerio de Cultura y Educacion de la Provincia de Córdoba (1994). Lineamientos curriculares para el nivel primario. Documento base para la adecuación a los contenidos básicos comunes y a la estructura del sistema según la Ley Federal de Educación. Córdoba: División de Tecnología Educativa.

Ministerio de Cultura y Educacion de la Provincia de Córdoba (1997). Propuesta curricular - Nivel Primario: $1^{\circ}$ y $2^{\circ}$ ciclo EGB. Córdoba: Dirección de Planificación y Estrategias Educativas.

Parra, Cecilia; Sadovsky, Patricia \& Saiz, Irma. (1994). Enseñanza de la Matemática. Documento curricular. Materiales de enseñanza destinados a la capacitación docente. Ministerio de Cultura y Educación de la Nación.

PARRA, Cecilia \& SAIZ, IRMA (comps). (1997). Didáctica de las matemáticas - aportes y reflexiones. Buenos Aires: Editorial Paidós Educador.

Pascual, Lidia; Candás, Selva y Fernández, Stella (1997a). Un vacío pedagógico: las tareas del cuaderno. En revista Zona Educativa. Ministerio de Cultura y Educación de la Nación. Año 2. Número 17, pp 55-57.

PAscual, Lidia; CANDÁs, Selva y Fernández, Stella (1997b). El cuaderno como recurso de evaluación. En revista Zona Educativa. Ministerio de Cultura y Educación de la Nación. Año 2. Número 18, pp 5557.

PólyA, George (1957). How to solve it: A new aspect of mathematical method. Garden City, New York: Doubleday.

SeCRETARIA MinISTERIO de EduCACIÓn (1987). Reforma educacional de Córdoba: Lineamientos curriculares para el nivel primario (versión preliminar). Córdoba: División de investigaciones e innovaciones educativas. 


\title{
Contactar
}

Revista lberoamericana de Educación

\author{
Principal OEI
}

